\title{
Attractors of a randomly forced electronic oscillator
}

\author{
Peter Ashwin, \\ Department of Mathematical and Computing Sciences, \\ University of Surrey, \\ Guildford GU2 5XH, UK.
}

September 23, 1998

\begin{abstract}
This paper examines an electronic oscillator forced by a pseudo-random noise signal. We give evidence of the existence of one or more random attractors for the system depending on noise amplitude and system parameters. These random attractors may appear to be random fixed points or random chaotic attractors. In the latter case, we observe a form of intermittent synchronization of the response of the system to the noise signal. We show how this can be understood as on-off intermittency in an extended system.
\end{abstract}

Keywords: Chaotic dynamics, electronic oscillator, randomly forced system.

\section{Introduction}

Although one would like to model real systems in terms of deterministic systems, inevitably noise will be present from a number of sources. One can classify many influences that cannot be modelled in a deterministic way as random fluctuations; for example, thermal noise in electronic systems can be explained in terms of kinetic noise of electrons, but modelling a circuit by the motion of individual electrons is clearly no way forward. Thus, one tries to identify statistical properties of the noise (such as its variance) to model such influences as for example a stochastic differential equation. By doing this, one can investigate the statistical properties of the solution of the system. However, one may lose details of what might be called the 'fine-scale structure' of the motion.

Random dynamical systems (or RDS) have been studied over the last few decades as a way of modelling dynamical systems subject to the influence of added noise. In a random dynamical system, the noise process itself is modelled as a dynamical system whose statistical properties are known. It is particularly helpful in providing analytical tools appropriate for noise of any scale, not just for the low noise.

In this paper we examine how one can experimentally measure 'random attractors' and some of their properties (sensitivity to initial conditions) for pseudo-random forcing of an analogue electronic nonlinear oscillator of van der Pol-Duffing type. 
A random dynamical system is a skew product system where the evolution of the system after a time $t$ is given by a deterministic mapping

$$
\begin{aligned}
\omega(t) & =\theta_{t} \omega \\
y(t) & =\phi(t, \omega) y
\end{aligned}
$$

where $\theta_{t}$ is a flow and $\phi(t, \omega)$ is a cocycle (i.e. is such that $\phi(t+s, w)=\phi\left(t, \theta_{s} w\right) \circ$ $\phi(s, w)$ for all $t, s>0)$. $\omega$ represents the state of a dynamical system that models the noise process and $y$ represents the dynamical system forced by the noise. We write $\theta_{t} \omega(\operatorname{resp} . \phi(t, \omega) y)$ to mean a nonlinear map $\theta_{t}(\operatorname{resp} . \phi(t, \omega))$ applied to the point $\omega$ (resp. $y$ ). In particular, the action of $\theta$ and $\phi$ need not be linear. For a detailed discussion of theory for random dynamical systems, see the recent monograph by Arnold [3] or [2]; we will outline a few basic concepts and definitions below. In the case that the evolution $\theta$ is chaotic we can see the above system as random forcing of a deterministic system $\phi$ where the evolution of $\omega$ is 'hidden'. By looking at such systems one can get a more detailed picture of the dependence of a dynamical system on noise than is possible by, for example, a Fokker-Planck approach.

We assume that the evolution $\theta$ has an ergodic invariant probability measure $\mathcal{P}$ with respect to which an initial condition for $\omega$ is chosen from a set of full measure. Random dynamical systems studies the dynamics of the full system relative to the dynamics of the measure-preserving transformation $(\theta, \mathcal{P})(\mu$ is defined on subsets in some $\sigma$-algebra).

Many of the ideas from dynamical systems can be generalised to apply to RDS; for example a set can be generalised to a random set; this is a family of sets $A(\omega)$ that is defined for $\mathcal{P}$-almost all $\omega$. Such a set is invariant if $\phi(t, \omega) A(\omega)=A\left(\theta_{t} \omega\right)$ for almost all $\omega$. Similarly one can define random invariant measures and ergodicity of such measures.

The concept of a random attractor was introduced by Crauel and Flandoli [9] and Schenk-Hoppé [17]. We say $A(\omega)$ is a random attractor if it is a compact invariant random set such that there is a random domain of attraction consisting of a universe of random sets $B(\omega)$ with non-empty interior such that

$$
d\left(\phi\left(t, \theta_{-t} \omega\right) B\left(\theta_{-t} \omega\right), A(\omega)\right) \rightarrow 0
$$

as $t \rightarrow \infty$ for $\mathcal{P}$-almost all $\omega .{ }^{1}$ Note that this is a limit as time goes forwards for $\phi$, but we need to 'run the noise backwards' to ensure we are comparing like with like. As noted in [9], this implies that

$$
d\left(\phi(t, \omega) B(\omega), A\left(\theta_{t} \omega\right)\right) \rightarrow 0
$$

in a weaker sense of convergence (namely, convergence in probability) as $t \rightarrow \infty$. Using the backward limit (1) it is possible to show existence of e.g. global attractors and to construct them by looking at limits of the form

$$
\bigcap_{T \geq 0} \overline{\bigcup_{t \geq T} \phi\left(t, \theta_{-t} \omega\right) K}
$$

\footnotetext{
${ }^{1} d(A, B)=\sup _{a \in A} \inf _{b \in B}|a-b|$ is the Hausdorff semi-metric, and $d(A, B)=0$ implies that $A \subset \bar{B}$.
} 
for some non-random set $K$. Using (1) to check attraction to a random attractor is not possible in reality (one needs to run the noise process backwards), but nonetheless one can see evidence of attraction in the sense of (2).

In a previous paper [7] we consider how graphs of random attractors can in certain cases be seen as Milnor attractors [12] for the skew product system. We will be interested in minimal random attractors [7], as these are the attractors that are seen as $\omega$-limit sets of individual initial conditions.

A random attractor $A$ is a random fixed point if $A(\omega)$ is a point for almost all $\omega$. Typically $A(\omega)$ will have nontrivial dependence on $\omega$. However, for multiplicative noise, $A(\omega) \equiv 0$ will be a random fixed point.

There will be random invariant measures supported on the random attractor, as discussed in $[9,17]$, and it is possible to define Lyapunov exponents (LEs) on the random attractor with respect to these invariant measures. These characterize the motion of points near the attractor. These Lyapunov exponents are important in understanding bifurcations of random attractors [3]. In particular, if the LEs are all negative, the attractor will be a random fixed point. If one of the LEs of a random attractor is positive, we say it is a random chaotic attractor.

For the van der Pol-Duffing oscillator forced by pseudo-random noise, we observe the existence of one or more random attractors in the system. These can be "slaved" to the random forcing (attracting random fixed points) or they can be random chaotic attractors. Section 2 describes the experiment and some of the effects of randomly forcing the circuit at different parameter values. Note that similar graphs are studied for the chaotic forcing of systems discussed by [10, 18].

The random attractors have the structure of graphs over the state space of the random forcing; in Section 3 we measure some of these graphs. These graphs may be point valued or set valued. If the graph is set valued then there can be sensitive dependence on initial conditions for identical realizations of the noise. We observe intermittent deviations of the response of identical realizations from each other. In section 4 we study this in more detail and show how this can be viewed as an on-off intermittent synchronization effect and why we expect this to be a typical effect on forcing hyperbolic stable fixed points and limit cycles by i.i.d. noise.

\section{Forcing an electronic oscillator}

The oscillator of the simple van der Pol-Duffing type shown in Figure 1; it consists of two integrators, an inverter, a nonlinear resistance provided by a network of diodes and associated feedback components. The two variable resistors can be used to set parameter values $r_{1}$ and $r_{2}$ which unfold a codimension two Bogdanov-Takens

point. This is the intersection of a line of pitchfork bifurcations and a line of Hopf bifurcations of a trivial rest state. The offset nulls of the operational amplifiers were adjusted to ensure that the circuit is as symmetric as possible.

Note that the normal form of the Bogdanov-Takens bifurcation is precisely the van der Pol-Duffing equation

$$
\ddot{v}+\left(\mu-v^{2}\right) \dot{v}+\left(\nu-v^{2}\right) v=0
$$


after a suitable nondimensionalisation, and so although the nonlinear $I(V)$ response of the diodes is not cubic, the cubic terms will dominate near the Bogdanov-Takens point.

The circuit allows for setting of initial conditions by variable resistors and switching of the integrators by CMOS switches. It also includes an analogue forcing input as shown in Figure 1. The forcing and data capture was performed by interfacing a laptop $\mathrm{PC}$ with $\mathrm{A} / \mathrm{D}$ conversion hardware. If we non-dimensionalise the equations of motion for the circuit then we get the following ODE governing the evolution of the voltage $v_{1}$, where time is in units of $R C=0.1 \mathrm{mS}$.

$$
\ddot{v}_{1}+\left(\beta+3 K v_{1}^{2}\right) \dot{v}_{1}+\left(\alpha+K v_{1}^{2}\right) v_{1}=N(t)+\dot{N}(t)
$$

In (3) it can be shown that $\alpha=\frac{r_{2}-r_{1}}{\left(r_{1}+0.5\right)\left(r_{2}+0.5\right)}$ and $\beta=\frac{r_{2}-0.5}{r_{2}+0.5}$. The parameters $r_{i} \in$ $[0,1]$ represent the proportion of the full scale resistances of $R_{i} . K>0$ represents the rate of saturation of the diodes. $N(t)$ is the high pass filtered noise input $V_{i n}$; this has zero mean and its RMS amplitude can be set using the variable resistor $r_{3} \in[0,1]$ (arbitrary units).

The noise-free trivial solution $v_{1}=0$ of $(3)$ with $r_{3}=0$ loses stability via a Hopf bifurcation at $r_{2}=0.5$ for $r_{2}>r_{1}$ and by a pitchfork bifurcation at $r_{1}=r_{2}$; thus there is a codimension two Bogdanov-Takens bifurcation at $r_{1}=r_{2}=0.5$ approximately. In practice, the readings $r_{i}$ were taken from 10 turn potentiometers that were not calibrated; this means that the actual bifurcations occurred a few percent away from their predicted values.

The random digital signal was generated in software by iteration of a logistic map and outputting the most significant bit while simultaneously performing data capture from the circuit. That is, the iteration $x_{n+1}=4 x_{n}\left(1-x_{n}\right)$ was performed in double precision with $x_{0}=0.1$ and $V_{i n}$ was set to $0 \mathrm{~V}$ or $5 \mathrm{~V}$ according to whether $x_{n}>0.5$ or $x_{n}<0.5$. This is equivalent to an unbiased Bernoulli shift on sequences of two symbols. In fact, the forcing sequence is eventually periodic. However, even for 32 bit representation of the numbers, there will be orbits with period of order $2^{31}$ giving a repeat only after a time of order many days.

The analogue data capture rate for all the experiments was $10 \mathrm{kHz}$ while the random signal was clocked at a rate of $1.406 \mathrm{kHz}$, close to the natural oscillation frequency of the circuit. As the timing was done by a free-running program, interrupts to the processor very occasionally caused glitches in the timing that were obvious in the recorded timeseries. These could be easily spotted as a slight phase-shift on different runs with the same noise realization and hence removed.

Figure 2 shows an example of a phase portrait reconstructed from the randomly forced system by using delay coordinates. In the absence of noise, these parameters show a stable limit cycle surrounding three unstable equilibria.

\subsection{Results}

We now give some examples of timeseries that are typical for some runs using a number of parameter values and initial conditions. 


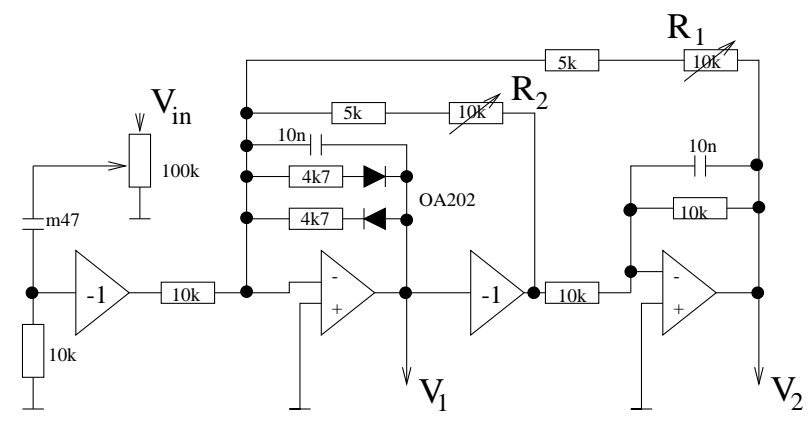

Figure 1: Schematic diagram of the analogue electronic experiment, a van der PolDuffing type oscillator with input for a random forcing signal. The variable resistors $R_{1}$ and $R_{2}$ control the unfolding of a codimension two Bogdanov-Takens point with symmetry. The measurable voltages $V_{1}$ and $V_{2}$ determine the state of the circuit and the input is via $V_{\text {in. }}$. There is extra circuitry (not shown) to allow one to charge the capacitors to specified voltages and then release the circuit from the given initial condition. All timeseries are measured at the point $V_{1}$.

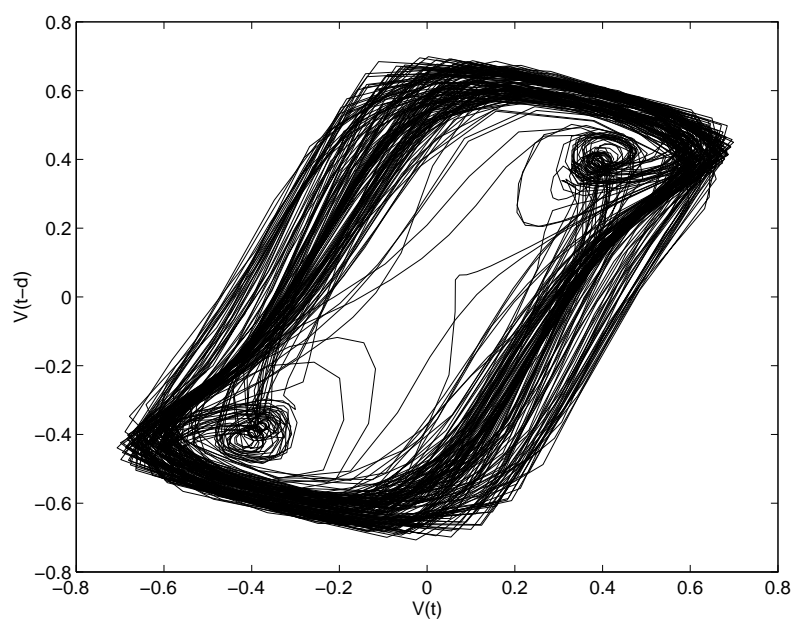

Figure 2: Phase portrait of the response $v_{1}(t)$ plotted against the delay $v_{1}(t-\tau)$ for $\tau=0.2 m S$ for $r_{1}=0.229, r_{2}=0.231$ and noise level $r_{3}=0.01$. Note that the trace is approximately following a noisy version of the a cycle that exists in the case of no noise $\left(r_{3}=0\right)$. 
Figure 3(a) shows the evolution of the same initial condition started five times using different realizations of the noise cycle for $r_{1}=0.26, r_{2}=0.231$ and $r_{3}=0.005$. Note how the effect of the noise determines whether the trajectory evolves to one or other of a pair of approximately fixed points. As we expect for different uncorrelated realizations of the noise, there is apparently no correlation between the fluctuations of the five trajectories.

Figure 3(b) shows the trajectories resulting from four different initial conditions responding to the same realization of the noise signal (shown at the top of the figure). There are two random attractors close to the symmetrically placed fixed points of the noise-free system and each has a basin of attraction. The fact that all initial conditions settle down to just one trajectory in each attractor implies that these random attractors are random fixed points.

This is shown more clearly in Figure 4(a) where four different initial conditions are shown in their response to the same realization of the noise. Note that one of them is released just after time step 7375; it spirals around both fixed points before becoming trapped near one of them and synchronizing with it. Figure 4(b) shows the difference between the evolution of two initial conditions subjected to the same noise realization. Observe that they are strongly synchronized.

At different values of the system parameters, namely $r_{1}=0.229, r_{2}=0.231$ and $r_{3}=0.01$ we see noise forcing of a limit cycle. Figure 5 shows random perturbations of a limit cycle for four different initial conditions (lower traces) and the forcing square wave (upper trace). Note that although the responses are strongly synchronized for long periods of time, there are time intervals where they diverge and their difference grows to the same order as the amplitude of the fluctuations. This is shown more clearly in Figure 6 where the difference between two of the runs for the same realization of the noise is shown in the bottom plot; in contrast to Figure $4(\mathrm{~b})$ there are many irregular bursts where the difference grows to the same order of magnitude as the signal.

Finally, Figure 7 shows the response of four different initial conditions to the same realization of the noise at parameter values $r_{1}=0.15, r_{2}=0.55$, and $r_{3}=0.01$. In the noise-free case the system has a fixed point near 0 that is near a Hopf bifurcation at $r_{2}=0.53$. This case shows evidence of an attracting random fixed point.

\section{Random attractors}

Although we cannot measure random attractors directly, we can measure indicators that imply their existence, multiplicity and whether they are random fixed points or not. If there is a minimal attractor that is a random fixed point then we will see convergence of a range of initial conditions in the sense of (2) to some unique 'chaotically varying' signal, regardless of initial condition. The sense of convergence may be quite weak (in probability), but in this experiment we only observed either convergence or clear non-convergence to a random fixed point. Non-convergence of an ensemble of initial conditions to any single trajectory is a feature of a random attractor that is chaotic. 


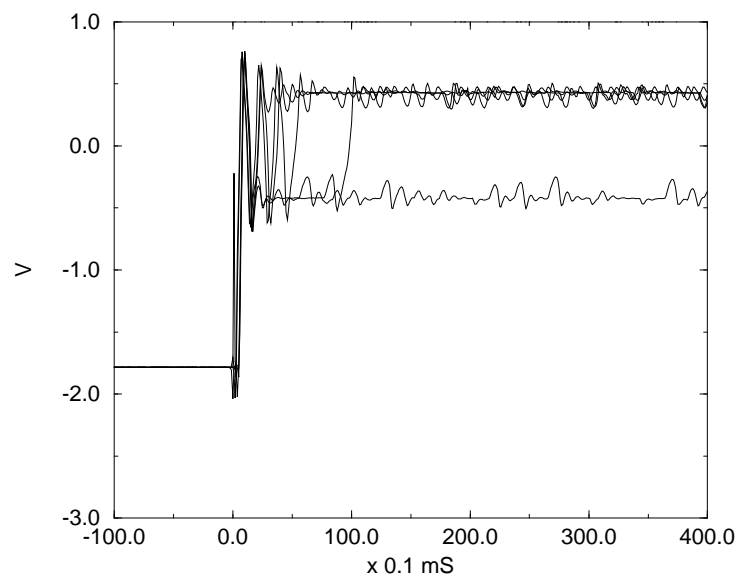

(a)

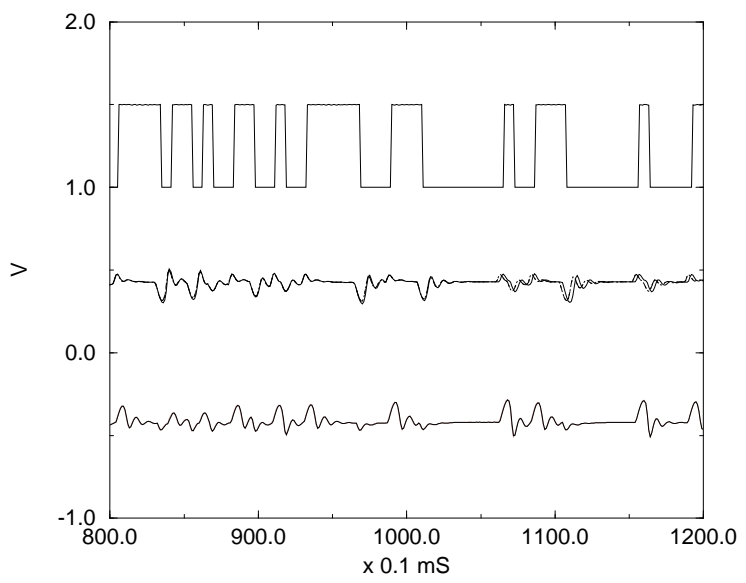

(b)

Figure 3: (a) Responses to five different realizations of the noise are shown for the system released from the same initial condition at time approximately zero, with $\left(r_{1}, r_{2}, r_{3}\right)=(0.26,0.231,0.005)$. Observe how all trajectories evolve to one of two approximately fixed points. (b) Four different initial conditions subjected to the same realization of the noise are shown for the system. Three trajectories are attracted to the upper random fixed point whereas the other trajectory evolves to a lower fixed point. The top line shows the random forcing square wave. 


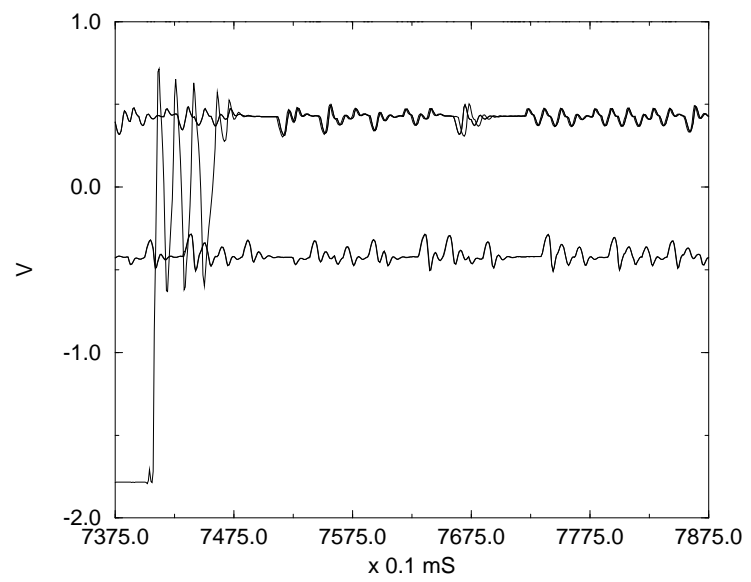

(a)

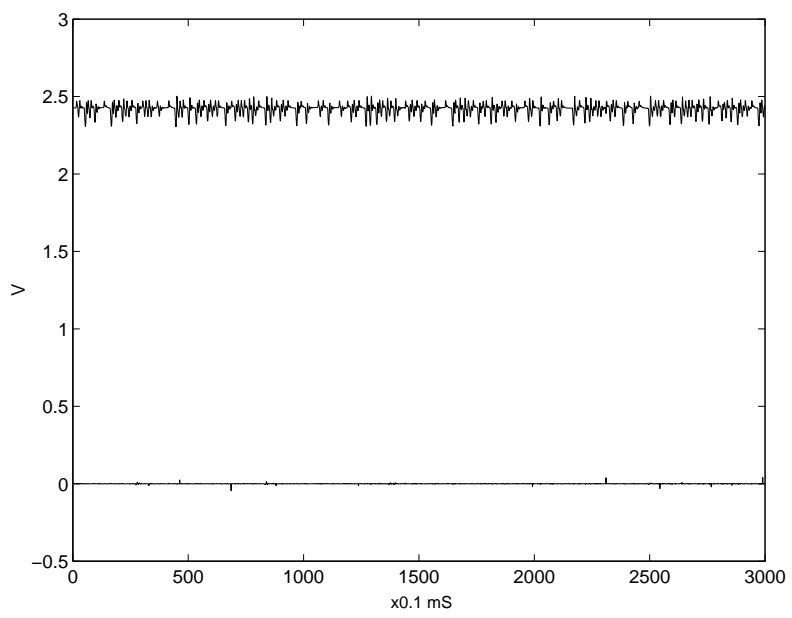

(b)

Figure 4: (a) A segment of the four time series in Figure 3(b) on which the evolution of an initial condition started just after sample 7375 is superimposed. Note how an initial transient oscillation results in the trajectory being attracted to the upper random fixed point attractor. (b) The bottom trace shows the difference in response of two initial conditions to the same realization of the noise for two sets of the data shown in Figure 3(b). The top curve shows one of the time series shown displaced for reference. The difference remains very small, indicating that the two timeseries have synchronized. 


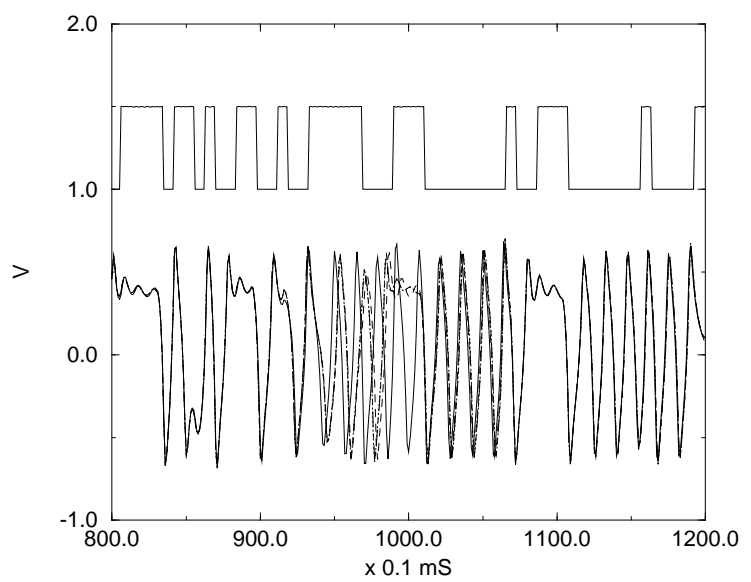

Figure 5: Four different initial conditions subjected to the same realization of the noise are shown, with $\left(r_{1}, r_{2}, r_{3}\right)=(0.229,0.231,0.01)$. All trajectories are attracted to an initial condition on a random limit cycle, though note that there seem to be occasional desynchronization events, for example just before sample 1000. The top line shows the random forcing square wave.

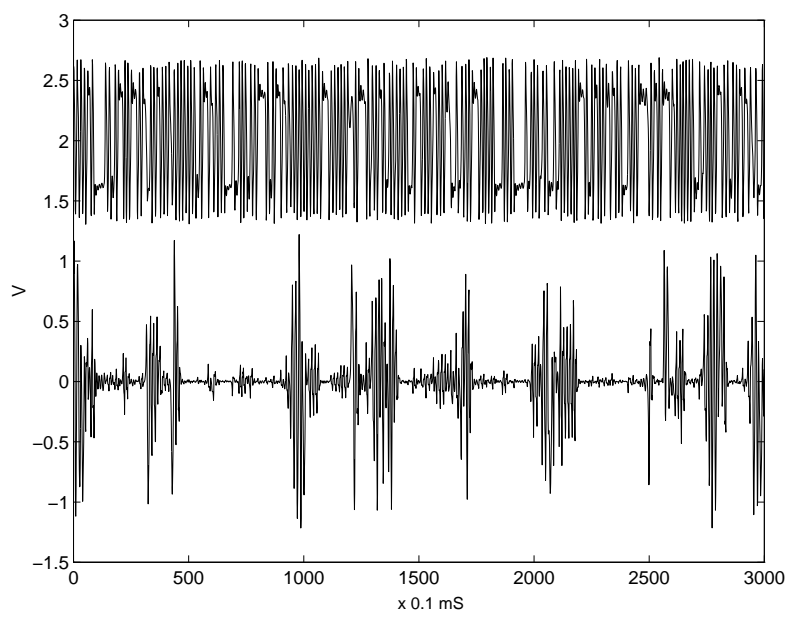

Figure 6: The bottom trace shows the difference in response of two initial conditions to the same realization of the noise for two sets of the data shown in Figure 5. the top curve is one of the time series shown displaced for reference. Observe the intermittent burstings of the difference; this can be understood as a form of on-off intermittency. 


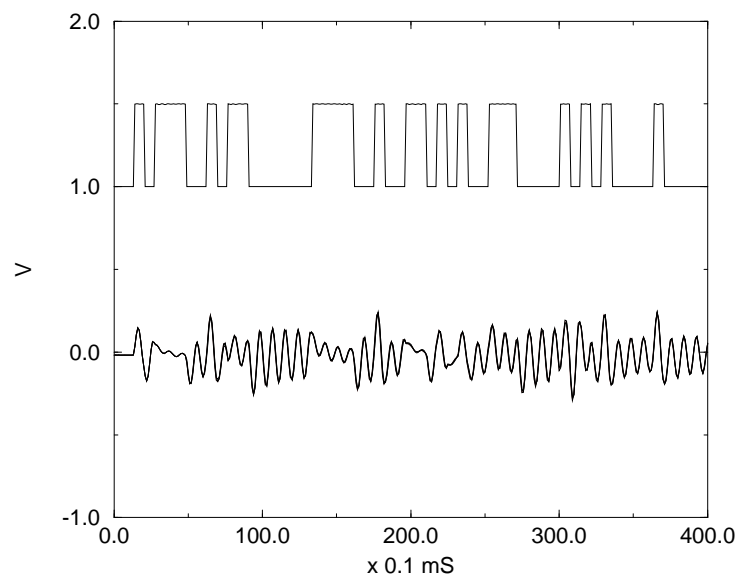

Figure 7: In this case $\left(r_{1}, r_{2}, r_{3}\right)=(0.15,0.55,0.01)$ and the bottom trace shows the superimposed evolutions of four different initial conditions subjected to the same realization of the noise, after transients have died away. The four traces are indistinguishable from each other. In this case the noise-free system evolves towards a single stable fixed point at 0 that is close to Hopf bifurcation. The top line shows the random forcing square wave (displaced for clarity).

More precisely, we measure the output $v_{1}(t)=V_{\text {ref }}(t)$ of the system with a linearly stable fixed point forced by the particular realization of the noise and parameter value. We can then measure dependence of the system in other parameter regimes by plotting the output against this reference.

Figure 8 shows the dependence of the system response on $V_{\text {ref }}(t)$ for a few different parameter values. Note that for $(\mathrm{a}-\mathrm{d})$ the response is strongly correlated with $V_{\text {ref }}(t)$ while for $(\mathrm{e}-\mathrm{h})$ it is not. We interpret plots (a-d) as the response being a projection of smooth graph of the reference signal onto one dimension and conclude that for all of these parameter values there is a random fixed point attractor that is varying continuously on changing the parameter.

The plots in (e-h) of this figure shows the evolution of four initial conditions at the same parameter value, subject to the same noise realization and plotted against $V_{\text {ref }}$ corresponding to the first response. The response always makes large deviations from the diagonal and does not appear to be restricted to any graph, indicating that the random attractor is not a random fixed point, but a random chaotic atttractor.

\section{On-off intermittency of differences}

Suppose that the system is governed by a differential equation

$$
\begin{aligned}
& \dot{x}=f(x) \\
& \dot{y}=g(x, y) .
\end{aligned}
$$





$(\mathrm{a}-\mathrm{h})$

Figure 8: Graphs of the response $V(t)$ agains a the reference response $V_{\text {ref }}(t)$ for $(a)$ (d) forcing of a stable fixed point on changing a parameter. Note how the presence of a high degree of structure in the cloud of points suggests that the random attractors in these cases are random fixed points. The forcing amplitude is $r_{3}=0.01$ in all cases and $\left(r_{1}, r_{2}\right)=(a)(0.1,0.9)$, (b) $(0.2,0.9)$, (c) $(0.3,0.9)$, (d) $(0.5,0.9)$. For (e)-(h) the forcing is of a limit cycle at $\left(r_{1}, r_{2}\right)=(0.229,0.231)$ with $r_{3}=0.01$. The four panels show the graph of four realizations $V$ plotted against the first realization $V_{\text {ref }}$ in panel (e). 
We consider the evolution of two points $y_{0}$ and $y_{1}$ under the same realization of the noise $x(t)$. We can view this as as a 'double skew product':

$$
\begin{aligned}
& \dot{x}=f(x) \\
& \dot{y}_{1}=g\left(x, y_{1}\right) \\
& \dot{y}_{2}=g\left(x, y_{2}\right)
\end{aligned}
$$

where the two runs are performed at different times using the same noise. The extended system (5) has a $\mathbf{Z}_{2}$ symmetry given by $\left(x, y_{1}, y_{2}\right) \mapsto\left(x, y_{2}, y_{1}\right)$ and in particular it has an invariant subspace given by $y_{1}=y_{2}$ and no restriction on $x$; we refer to this as the synchronized subspace and note that there is a fairly complete theory describing the transverse stability and instability of an attractor in such a synchronized subspace. However, in this case there is a special structure (no coupling between $y_{1}$ and $y_{2}$ ) that means we can say more about this system.

Note that (5) has been studied by Abarbanel et al. [1] and their concept of generalised synchronization is equivalent to existence of a random fixed point attractor for the RDS. It can also be seen an example of the synchronizing systems considered by Pecora and Carroll [14] who allow coupling from $y_{1}$ to the $x$ system but not from $y_{2}$. In their terminology the system $\left(x, y_{1}\right)$ is a master system and $y_{2}$ the slave. In our case $\left(x, y_{2}\right)$ could also be considered to be the master system, given the symmetry. The random attractors for the system (5) are also important for determining the so-called two-point motion of the original RDS (4) [8].

Let $\lambda_{i}, i=1 . . n$ be the conditional Lyapunov exponents of the system (4) corresponding to the growth of perturbations in the directions $y$ for forcing (with respect to the assumed natural measure for attractor of the dynamics of (4)). It is easy to see that (5) will have two copies of each of these Lyapunov exponents, one copy being tangential Lyapunov Exponents inside the synchronized subspace and the other

being transverse Lyapunov exponents corresponding to desynchronizing perturbations.

Recall that the value of Lyapunov exponents depends not just on an invariant set but more precisely on the invariant measure supported on that set. Moreover, any chaotic attractor will support a set of invariant measures corresponding to unstable invariant sets within the attractor; this will cause the existence of a spectrum of LEs for the forcing chaotic dynamics $x$.

\subsection{A scenario for on-off intermittency of differences}

Suppose that the system (5) has a unique random attractor with $\lambda_{1}>0$ and that there is an (unstable) fixed point $f\left(x_{0}\right)=0$ in the attractor for the $x$ dynamics. Suppose also that there is a unique quiescent (noise-free) attractor of

$$
\dot{y}=g\left(x_{0}, y\right)
$$

that is a hyperbolic stable fixed point. Then we expect the random attractor for (5) to display on-off intermittency [15], i.e. arbitrarily long periods in any neighbourhood of the synchronized subspace interspersed with large deviations away from it. 
The presence of the fixed point $x_{0}$ means there is a singular measure for the dynamics of the random attractor where the conditional LEs $\lambda_{i}$ are all negative. Since the transverse LEs for the synchronized state $y_{1}=y_{2}$ are copies of the conditional LEs for $y_{1}$, there will be a positive transverse LE and so we can get on-off intermittency as noted in [6].

Alternatively, note that any typical trajectory $\theta_{t} w$ will spend arbitrarily long times near $x_{0}$. During these times, the hyperbolic fixed point will be attracting and the synchronized state attracting. At other times (and indeed on average) the existence of a positive conditional LE will force trajectories of (5) away from the synchronized state and hence an intermittent bursting will be evident.

In fact, due to bubbling [5] we do not expect the existence of a positive conditional Lyapunov exponent for the natural measure is necessary. In the presence of random perturbations to the $x$ dynamics, a weaker assumption that there is a positive conditional L.E. for any measure supported on the random attractor should suffice to give instability of the synchronized state for (5). This will be the case in the experiment where there is real noise present at a lower amplitude than the pseudo-random signal.

Note that the assumption of a unique random attractor would preclude the possibility of a random attracting fixed point coexisting with the random chaotic attractor. A more reasonable assumption might be to assume local uniqueness of the attractor, i.e. existence of a random neighbourhood of the random attractor where the attractor is unique.

\section{Discussion}

This investigation was motived by Schenk-Hoppé's work [16] on a random dynamical systems interpretation of the stochastic van der Pol-duffing oscillator; his work is primarily analytical and numerical and has highlighted how one can see stochastic D-bifurcations as being instrumental to understanding qualitative changes in the stochastically forced system on changing parameters.

The electronic system we have discussed allows fast and easy experimentation with a similar system, a van der Pol-Duffing type nonlinear oscillator with a form of noise forcing. We have not attempted to map out the parameter plane but instead have focussed on some examples of behaviour on random attractors. We show that these random attractors may or may not be random fixed points; in the case that they are not we have observed an associated on-off intermittency of differences.

To summarise some new features in comparison to $[4,16]$

(A) We have found random chaotic attractors. These were not found in $[4,16]$, possibly due to the form of noise (white noise) and possibly due to the investigation there concentrating on the bifurcation properties of the system. However, more recent numerical work [11] indicates that random chaotic attractors are observable in this system.

(B) We have found example of bistability (coexistence of two random attractors). 
This was not found in [16] due to the unboundedness of the white noise.

Of course, the qualitative and quantitative response of the system depends strongly on the fact that we are using finite amplitude noise that has a particularly simple form. If we were to consider white noise forcing we would find that any random attractor will be typically unbounded owing to the very small but finite probability that the noise can force a jump in response of any size. This will also mean that there can only be one random attractor and so bistability of random attractors cannot occur (moreover it implies that we cannot expect convergence to random attractors in the sense of (2) for almost all $\omega$ ).

As we have demonstrated, the system lends itself to an examination of random attractors in a physical system. There are other aspects that could be examined using this setup; for example it is possible to control the forcing output to model dynamics that are contained within the attractor generating the chaotic signal. In this way we may be able to understand the transition to on-off intermittency of differences discussed in Section 4 in terms of a blowout bifurcation [13] of the extended system (5). We leave this for future study.

\section{Acknowledgements}

I thank the Nuffield foundation for support in the form of a "Newly appointed science lecturer" grant, and Alan Maclean for help with the electronics. I also thank Ludwig Arnold, Hans Crauel and Klaus Reiner Schenk-Hoppé for some very interesting conversations on the subject of random dynamical systems. I thank a referee for bringing my attention to [1].

\section{References}

[1] H.D.I. Abarbanel, N.F. Rulkov and M.M. Sushchik. 1996. Generalized synchronization of chaos: the auxiliary system approach. Phys. Rev. E, 53:45284535 .

[2] L. Arnold. 1995. Six lectures on random dynamical systems. In: R. Johnson, ed, Dynamical Systems, Volume 1609 of lecture notes in mathematics, pp 1-43, Springer Verlag Berlin.

[3] L. Arnold. 1997. Random Dynamical Systems (preprint).

[4] L. Arnold, N. Sri Namachchivaya and K.R. Schenk-Hoppé. 1997. Toward an understanding of stochastic Hopf bifurcation: a case study. International Journal of Bifurcation and Chaos 6:1947-1975.

[5] P. Ashwin, J. Buescu and I.N. Stewart. 1994. Bubbling of attractors and synchronisation of oscillators. Phys. Lett. A 193: 126-139. 
[6] P. Ashwin, J. Buescu and I.N. Stewart. 1996. From attractor to chaotic saddle: a tale of transverse instability. Nonlinearity 9:703-737.

[7] P. Ashwin. 1997. Minimal attractors for random dynamical systems Technical Report, Dept of Maths and Stats, University of Surrey. Submitted to Proc Roy Soc Lond. A.

[8] P. Baxendale. 1996. Stability and equilibrium properties of stochastic flows of diffeomorphisms. In: Diffusion processes and related problems in analysis, Vol II; stochastic flows. Eds M. Pinsky and V. Wihstutz. Progress in Probability, 27: 3-35 Birkhäuser, Boston.

[9] H. Crauel and F. Flandoli. 1994. Attractors for random dynamical systems. Prob. Theory and Related Fields 100:365-393.

[10] M. Davies and K. Campbell. 1996. Linear recursive filters and nonlinear dynamics. Nonlinearity 9:487-499.

[11] H. Keller and G. Ochs. 1998. Numerical approximation of random attractors. Preprint, Institute for Dynamical Systems, University of Bremen.

[12] J. Milnor. 1985. On the concept of attractor. Commun. Math. Phys. 99:177195 Comments 1985 Commun. Math. Phys. 102:517-519.

[13] E. Ott, J.C. Sommerer. 1994. Blowout bifurcations: the occurrence of riddled basins and on-off intermittency. Phys. Lett. A 188:39-47.

[14] L.M. Pecora and T.L. Carroll. 1990. Synchronization in chaotic systems. Phys. Rev. Lett. 64:821-824.

[15] N. Platt, E.A. Spiegel and C. Tresser. 1993. On-off intermittency; a mechanism for bursting. Phys. Rev. Lett. 70:279-282.

[16] K.R. Schenk-Hoppé. 1996. Stochastic Hopf bifurcation: an example. Int. J. Non-Linear Mechanics 31:685-692.

[17] K.R. Schenk-Hoppé. 1998. Random Attractors - general properties, existence and applications to stochastic bifurcation theory. Discrete and Continuous Dynamical Systems 4:99-130.

[18] J. Stark. 1997. Invariant Graphs for Forced Systems Physica D 109: 163-179. 